\title{
Long Period Waveguide Gratings in Planar Gradient Waveguides
}

\author{
T. KOTYCZKA ${ }^{a}$ AND R. ROGOZIŃSKI ${ }^{b, *}$ \\ ${ }^{a}$ WASKO S.A., Berbeckiego 6, 44-100 Gliwice, Poland \\ ${ }^{b}$ Optoelectronics Department, Silesian University of Technology \\ B. Krzywoustego 2, 44-100 Gliwice, Poland
}

\begin{abstract}
This paper introduces the application possibilities of long-period waveguide gratings in planar structures of gradient waveguides. The analysis of resonance possibilities of coupling modes in gradient-gradient structures was carried out. An original configuration of single-mode (gradient) structure and multimode decoupling area was proposed. The refractive properties of gradient areas were calculated on the basis of experimental data relating to the $\mathrm{Ag}^{+} \leftrightarrow \mathrm{Na}^{+}$ion exchange in soda-lime glass. The calculations of the resonance properties included the actual dispersion of the glass substrate. The influence of the ion exchange technological processes (diffusion, heating) on the character of the coupling modes' curves was also presented. The calculations indicate the possibility of obtaining these resonances in the wavelength range corresponding to the visible spectrum.
\end{abstract}

PACS: 81.20.Fw, 42.70.Ce, 07.07.Df, 42.79.Gn

\section{Introduction}

The long-period gratings produced in waveguide structures enable the power coupling between the propagating wave modes. The long-period gratings in fiber waveguides (LPFGs) in optical fibers function as separate areas, in which periodic corrugations of permittivity of medium were produced. Those corrugations can be produced in the whole waveguide's cross-section or in its border area. The long-period gratings' structures were originally applied in the fiber optics in reference to the fiber waveguides [1]. They are realized by creating changes in the refractive index of the core of single-mode fiber. The techniques used for producing the Bragg gratings (e.g.: the waveguide core of silica glass doped with $\mathrm{GeO}_{2}$ then subjected to high-pressure exposure $\mathrm{H}_{2}+$ deep UV radiation - obtained refractive changes $\approx 10^{-3}[2]$ ), are also used for this purpose. The LPFGs implement coupling of the basic mode's optical power to the cladding modes. This coupling has a resonant character and the resonance wavelength for it is determined by the corrugation period and propagation constants of coupled modes. Those resonances for single-mode fiber waveguides are in the infrared range. LPFG are used as filters $[1,3-5]$ and a variety of sensory structures [6-8].

The possibility of long-period gratings usage in the structures of planar waveguides (LPWG) was demonstrated in [9]. The strip waveguide step-index type (epoxy novolak resin) formed on oxidized silicon sub-

* corresponding author; e-mail: roman.rogozinski@polsl.pl strate $\left(\mathrm{SiO}_{2}-\mathrm{Si}\right)$ was used there. The corrugation area of the silicon substrate surface was prepared by ion reactive sputtering prior to its oxidation. In such formed LPWG structure the resonant coupling modes in the wavelength range: $1280 \div 1550 \mathrm{~nm}$ and $-18 \mathrm{~dB}$ transmission peaks were obtained. In later works the LPWG structures were produced on substrates including glass, in which the planar waveguides were obtained by ion exchange method. In [10], the authors used for this purpose BK7 glass doped with silver ions $\left(\mathrm{Ag}^{+}\right)$. On the surface of the waveguide a corrugated area was produced (ion reactive sputtering). This area was then covered with a $4 \mu \mathrm{m}$ thick polymer layer with lower refractive index than the waveguide formed in the glass. This layer acted as a cladding (the equivalent in fiber waveguide), which could promote leaky modes. In such structures the resonant coupling in the wavelength range $1590 \div 1620 \mathrm{~nm}$ were obtained.

In the literature we can find descriptions of theoretical LPWG structures' usage in the step-index waveguide systems: (1D) planar slab waveguides $[11,12]$ as well as (2D) ridge waveguides $[13,14]$. The attractiveness of such structures results from the high flexibility of choice of materials and technologies used in their production. The possibilities are far greater than that of the LPFG structures. The choice of materials, which produce both the same fiber as well as the decoupling layer, allows the formation of $\lambda_{\mathrm{r}}$ resonance wavelength dependence from the $\Lambda$ period corrugations. Resonance characteristics $\lambda_{\mathrm{r}}(\Lambda)$ may include here the visible spectrum range. It is also possible to influence the spectral transmission characteristics. These features determine the great interest in this type of structures. 
In this paper there were proposed the LPWG structures on the basis of the planar gradient waveguides. Particular novelty is the use of gradient multimode region formed in the glass substrate, which is equivalent to the decoupling layer. Such a solution according to our knowledge has not been previously discussed in the literature. The presented calculations regard planar waveguide structures produced by ion exchange of $\mathrm{Ag}^{+} \leftrightarrow \mathrm{Na}^{+}$in soda-lime glass. The choice of this glass was imposed by the knowledge of the temperature coefficients of diffusion of exchanged ions. These relationships were determined experimentally for the source of admixture in the form: pure $\mathrm{AgNO}_{3}$ and $\mathrm{NaNO}_{3}$ solution of $\mathrm{AgNO}_{3}$ in the mole fraction of $\mathrm{mf}=0.0025[9]$.

\section{The idea of the gradient waveguide-based LPWG structure}

The implementation of coupling modes in planar waveguides is achieved by creating a periodic corrugation (with a $\Lambda$ period) in the leading area of the wave. For practical reasons, coupling occurs between the primary mode $\left(\mathrm{TE}_{0}\right.$ or $\left.\mathrm{TM}_{0}\right)$ and the modes of higher order, which propagate within the coverage area of the waveguide. The $\Lambda$ corrugation period is associated with the $\boldsymbol{K}$ wave vector of the grid $|\boldsymbol{K}|=2 \pi / \Lambda$. The condition for coupling modes in such a structure is described by Bragg's equation

$$
\boldsymbol{\beta}_{0}+q \boldsymbol{K}=\boldsymbol{\beta}_{m}^{\mathrm{cl}},
$$

where $\boldsymbol{\beta}_{0}$ and $\boldsymbol{\beta}_{m}^{\mathrm{cl}}$ - propagation constants of zero and $m$-th order, $q$ - coupling order $(q= \pm 1, \pm 2 \ldots)$.

Since the propagation constants satisfy the relation: $\beta_{m}^{\mathrm{cl}}<\beta_{0}$, the coupling order has negative values $(q<0)$. For $q=-1$ Eq. (1) expressed in effective refractive indices of the modes has the form

$$
\left(N_{0}-N_{m}^{\mathrm{cl}}\right) \Lambda=\lambda_{\mathrm{r}}
$$

where $\lambda_{\mathrm{r}}$ is the resonant wavelength for the coupling modes of zero and $m$-th order.

Since the effective refractive indices of the modes $N_{0}(\lambda)$ and $N_{m}^{\mathrm{cl}}(\lambda)$ are of dispersion character, the refractive dispersion of the waveguide medium and the coverage area has also an important influence on Eq. (2).

Figure 1a presents the realization of the LPWG in the planar waveguide structure step-index type. Such a solution can be found in the literature [10-15]. The usage of the gradient waveguide $\left(\mathrm{K}^{+} \leftrightarrow \mathrm{Na}^{+}\right.$ion exchange in BK7 glass) can be found in [11]. However, in this case also the decoupling area is a homogeneous layer deposited on its surface. In Fig. 1b our idea of LPWG realization in gradient-gradient structure was shown. The single-mode gradient waveguide has corrugated surface in the form of relief. The multimode decoupling area arises as a result of the additional diffusion of admixture into the glass through the corrugation zone. In this case the decoupling area is in the glass substrate. Figure 2 presents the sequence of technological processes allowing to implement the structure of Fig. 1b.

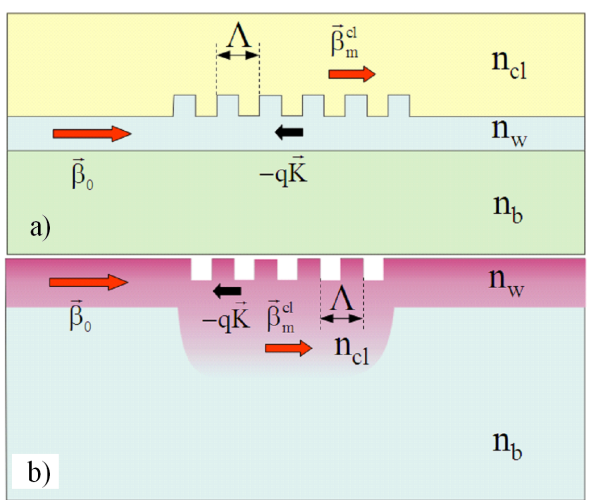

Fig. 1. The realization of LPWG in the planar waveguide structure: (a) step-index type, (b) proposed gradient-gradient structure.

The first technological step is to produce the corrugation (the relief) on a glass substrate (Fig. 2a). This is done by chemical etching (sputtering) of the glass prior to doping. In the next stage the selective ion exchange process through the glass surface (relief) is carried out (Fig. 2b). The resulting planar waveguide is a multi-mode structure. After this step, it is possible to have an optional heating step, which enables an additional diffusion of admixture introduced previously into the glass. As a result the refractive index profile of the waveguide is decreased, while increasing the range of admixture in the glass (increasing the depth of the waveguide) at the same time. This procedure allows the formation of the resonant coupling characteristics of the modes in the final LPWG structure.

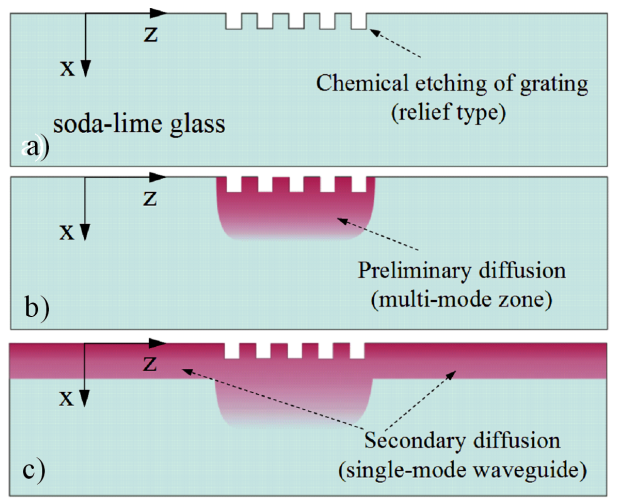

Fig. 2. The sequence of technological processes' realization of the structure in Fig. $1 \mathrm{~b}$.

The last technological step is to create a single-mode gradient waveguide across the whole surface of the glass substrate (Fig. 2c), which is achieved through the short process of secondary diffusion. In this process the refractive index of the multi-mode waveguide also increases. The duration of the secondary diffusion process must be chosen so that the resulting waveguide was single-mode 
throughout all of the used range of spectrum. This requires knowledge of the refractive dispersion of the glass substrate. Figure 3 presents the dispersion characteristics of the refraction of the soda-lime glass (own results).

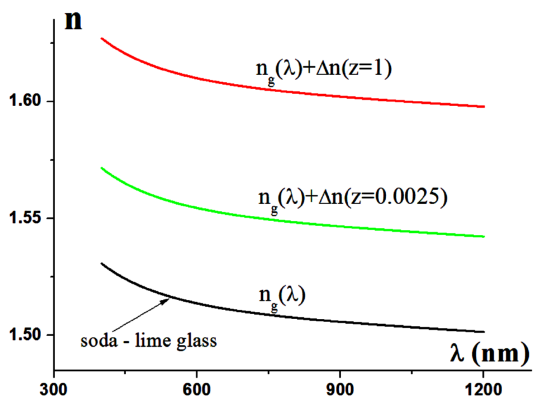

Fig. 3. Dispersion of the refractive index of soda-lime glass and its waveguide (admixture) area of $\mathrm{Ag}^{+}$ions in case of $\mathrm{AgNO}_{3}(z=1 \mathrm{mf})$ and $\mathrm{AgNO}_{3}$ in $\mathrm{NaNO}_{3}$ $(z=0.0025 \mathrm{mf})$ solution as admixture source.

Changes in refraction of the waveguide area were included assuming a constant refractive index increment $\Delta n$ at the glass surface (regardless of the wavelength). In Fig. 3, these relationships are shown with red line for the source of $\mathrm{AgNO}_{3}(z=1 \mathrm{mf})$ and green for a solution of $\mathrm{AgNO}_{3}$ in $\mathrm{NaNO}_{3}(z=0.0025 \mathrm{mf})$. The magnitude of these increments was determined experimentally [9]. In the case of ion exchange of $\mathrm{Ag}^{+} \leftrightarrow \mathrm{Na}^{+}$in soda-lime glass, they amount to, respectively, $\Delta n=0.0963$ for $z=1$ and $\Delta n=0.0408$ for $z=0.0025$. Still, in all analyses a spectral range of 400 to $1200 \mathrm{~nm}$ has been assumed.

\section{Analysis of the LPWG properties on the basis of gradient waveguides}

The assumptions adopted in the previous chapter have been used to analyze the LPWG structures produced by using the planar gradient waveguides. All calculations were made for soda-lime glass and admixture source of $\mathrm{AgNO}_{3}$ or $\mathrm{AgNO}_{3}$ solution in $\mathrm{NaNO}_{3}(z=0.0025 \mathrm{mf})$. Calculations of refractive index profiles resulting from diffusion and heating processes were conducted on the basis of model of the binary ion exchange. In this model a dependence of diffusion coefficients of exchanged ions from their normalized concentrations [16, 17] was adopted. Using this model the temperature dependences of diffusion coefficients of exchanged ions were experimentally determined. These dependences [18] for the $\mathrm{AgNO}_{3}$ admixture source are described by Eqs. (3):

$$
\begin{aligned}
& D_{A}(T)=\exp \left(-\frac{11085.8}{T}+19.9\right)\left[\frac{\mu \mathrm{m}^{2}}{\mathrm{~h}}\right], \\
& D_{B}(T)=\exp \left(-\frac{9298.8}{T}+19.1\right)\left[\frac{\mu \mathrm{m}^{2}}{\mathrm{~h}}\right] .
\end{aligned}
$$

The corresponding relations for diluted admixture sources of $\mathrm{AgNO}_{3}$ in $\mathrm{NaNO}_{3}(z=0.0025 \mathrm{mf})$ are described by Eqs. (4):

$$
\begin{aligned}
& D_{A}(T)=\exp \left(-\frac{8945.1}{T}+16.6\right)\left[\frac{\mu \mathrm{m}^{2}}{\mathrm{~h}}\right], \\
& D_{B}(T)=\exp \left(-\frac{43.9}{T}+3.5\right)\left[\frac{\mu \mathrm{m}^{2}}{\mathrm{~h}}\right] .
\end{aligned}
$$

In the above equations, the $D_{A}$ and $D_{B}$ are respectively: the diffusion coefficients of $\mathrm{Ag}^{+}$and $\mathrm{Na}^{+}$in the soda-lime glass. The temperature is in the absolute scale.

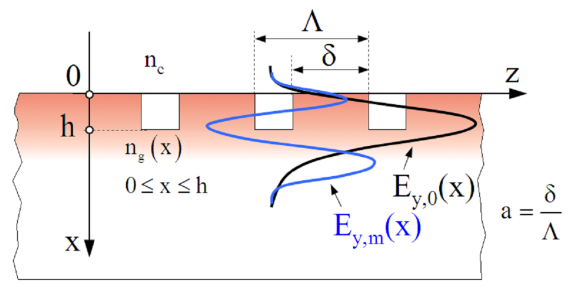

Fig. 4. Mode fields in the area of corrugated planar LPWG structure.

The waveguide area produced in the soda-lime glass by ion exchange of $\mathrm{Ag}^{+} \leftrightarrow \mathrm{Na}^{+}$has practically no birefringence. All calculations performed relate to the waveguide polarization of TE type. Calculated refractive index profiles were the basis for determining the dispersion dependences $N_{m}(\lambda)$ of the effective indices of guided modes. For the calculation the matrix method of planar waveguide analysis was used [19]. Based on these relationships the characteristics of the resonant coupling of modes $\mathrm{TE}_{0} \rightarrow \mathrm{TE}_{m}$ were determined by using Eq. (2).

Based on the $N_{m}(\lambda)$ dependence the spectral distributions of the fields of modes $E_{y 0}(x, \lambda)$ and $E_{y m}(x, \lambda)$ for the coupling areas of modes in LPWG structures was also calculated. Distributions of these fields allow calculations of the coupling coefficients of modes in the corrugated area [20]:

$$
\begin{aligned}
\kappa_{-1, \mathrm{TE}_{0} \rightarrow \mathrm{TE}_{m}}(\lambda)=\frac{1}{\lambda} \frac{\sin (a \pi)}{\sqrt{N_{0}(\lambda) N_{m}(\lambda)}} \\
\times \frac{\int_{0}^{h} E_{y, 0}(x, \lambda) E_{y, m}(x, \lambda)\left[n_{\mathrm{g}}^{2}(x, \lambda)-n_{\mathrm{c}}^{2}(\lambda)\right] \mathrm{d} x}{\sqrt{\int_{-\infty}^{\infty} E_{y, 0}^{2}(x, \lambda) \mathrm{d} x \int_{-\infty}^{\infty} E_{y, m}^{2}(x, \lambda) \mathrm{d} x}},
\end{aligned}
$$

where $\kappa_{-1}-$ coupling coefficient of the coupling row $(q=-1), a-$ duty-cycle of corrugation, $h$ - depth of corrugation, $n_{\mathrm{g}}(x, \lambda)$ - dispersion dependence of gradient area refraction in the glass, $n_{\mathrm{c}}(\lambda)$ - dispersion dependence of the refraction of the waveguide coverage.

Based on the specified coupling coefficients (5) the spectral transmission of the basic mode associated with the decoupling spectrum to the mode of $m$-th order is calculated [20].

$$
\begin{aligned}
& T_{\mathrm{TE}_{0} \rightarrow \mathrm{TE}_{m}}\left(\lambda, h_{\mathrm{g}}, a, \Lambda, L\right) \\
& =1-\frac{\sin ^{2}\left(\sqrt{\kappa_{-1, \mathrm{TE}_{0} \rightarrow \mathrm{TE}_{m}}^{2}+\Delta^{2}} L\right)}{1+\frac{\Delta^{2}}{\kappa_{-1, \mathrm{TE}_{0} \rightarrow \mathrm{TE}_{m}}^{2}}},
\end{aligned}
$$

where $L$ - length of corrugated area in the direction of 
propagation, $\Delta$ - a factor describing the deviation from phase matching according to Bragg's Eq. (1):

$$
\Delta(\lambda, \Lambda)=\pi\left(\frac{N_{m}(\lambda)-N_{0}(\lambda)}{\lambda}-\frac{1}{\Lambda}\right) .
$$

\subsection{Influence of the technological processes on the resonant coupling of modes}

A study was carried out to show the impact of technological processes related to the production of multimode decoupling area on the nature of the resonance curves $\lambda_{\mathrm{r}}(\Lambda)$. The processes of diffusion and heating have here major influence on the shape of refractive index profile.
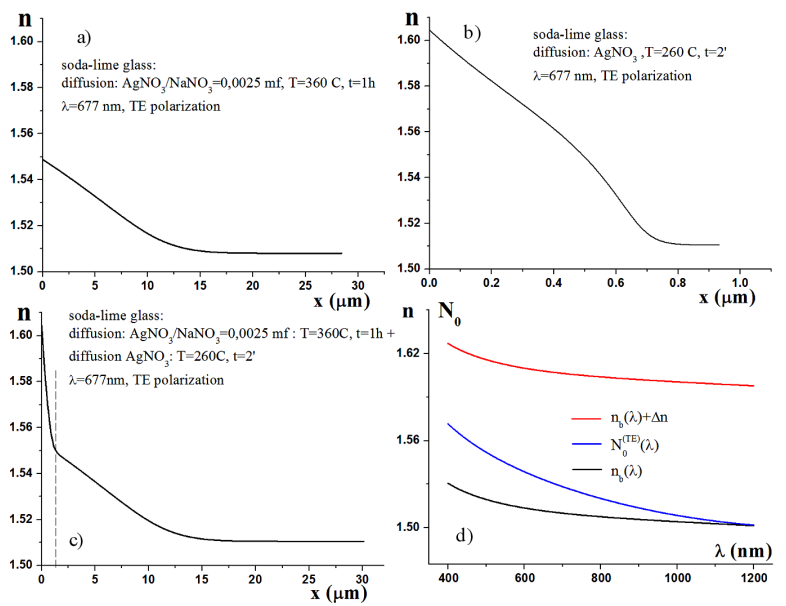

Fig. 5. The LPWG structure realized in two diffusion processes: (a) refractive index profile of the multi-mode area $(\lambda=677 \mathrm{~nm})$ after the preliminary diffusion $t=$ $1 \mathrm{~h}, T=360^{\circ} \mathrm{C}$, (b) refractive index profile of the single-mode area after the diffusion $t=2 \mathrm{~min}, T=260^{\circ} \mathrm{C}$, (c) refractive index profile of the multi-mode area covered by the secondary diffusion $t=2 \mathrm{~min}, T=260^{\circ} \mathrm{C}$, (d) dispersion of the glass substrate, glass doping area and the effective refractive index of $\mathrm{TE}_{0}$ mode in the wavelength range $400 \div 1200 \mathrm{~nm}$. All calculations are for soda-lime glass and the solution of $\mathrm{AgNO}_{3}$ in $\mathrm{NaNO}_{3}=0.0025 \mathrm{mf}$ as an admixture source.

The first simulation concerns the gradient structure produced in two stages of diffusion processes. The first stage is the diffusion from diluted sources in order to produce a multi-mode waveguide in the corrugated area. In Fig. 5a the calculated refractive index profile $(\lambda=$ $677 \mathrm{~nm}$ ) of the multi-mode area formed in the initial diffusion was shown. This process was simulated for the admixtures source of $\mathrm{AgNO}_{3}$ in $\mathrm{NaNO}_{3}=0.0025 \mathrm{mf}$ solution, diffusion duration $t=1 \mathrm{~h}$ and the temperature of the process $T=360^{\circ} \mathrm{C}$. The second stage is to produce a single-mode waveguide. For its production the diffusion process from the concentrated source (pure $\mathrm{AgNO}_{3}$ ) was simulated. Because the waveguide should be single-mode in the spectrum of $\lambda=400 \div 1200 \mathrm{~nm}$ a short process of diffusion is needed here. The time of this process needs to ensure single-mode for $\lambda=400 \mathrm{~nm}$, while the propagation of single-mode (without cutoff) for $\lambda=1200 \mathrm{~nm}$.
Large increase of refractive index in the case of such diffusion requires a short duration. For practical reasons (the actual implementation process), it is recommended to use possible low-temperature diffusion. This process was simulated for the temperature $T=260^{\circ} \mathrm{C}$, its duration is $t=2 \mathrm{~min}$. Figure $5 \mathrm{~b}$ shows the refractive index profile calculated for such a waveguide $(\lambda=677 \mathrm{~nm})$. In turn, in Fig. 5c the calculated refractive index profile in multi-mode area is shown. The calculations have been taken on a secondary diffusion which gives single-mode profile (Fig. 5b). Figure 5d shows dispersion characteristics of the glass substrate, the doping area of the glass and the effective refractive index of the $\mathrm{TE}_{0}$ fundamental mode. The figure shows the single-mode of waveguide in the wavelength range $400 \div 1200 \mathrm{~nm}$. For a multi-mode gradient area (Fig. 5c) the dependences were calculated according to dispersive modes $\mathrm{TE}_{0} \ldots \mathrm{TE}_{6}$. These relationships are shown in Fig. 6a.

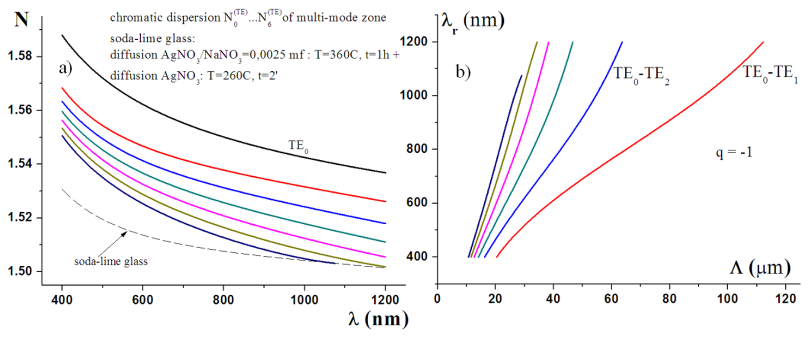

Fig. 6. (a) Chromatic dispersion of the effective refractive indices of the modes $\mathrm{TE}_{0} \ldots \mathrm{TE}_{6}$ of a multi-mode area (Fig. 4c). (b) The characteristics of resonant coupling of $\mathrm{TE}_{0}$ mode with $\mathrm{TE}_{1} \ldots \mathrm{TE}_{6}$ modes in a multi-mode area.

Figure $6 \mathrm{~b}$ shows the characteristics of the resonant coupling of $\mathrm{TE}_{0}$ mode with the modes of higher orders. These couplings occur in the multi-mode area (Fig. 5c). They were calculated on the basis of Eq. (2).

Two other ways of achieving LPWG structures are shown in Fig. 7, which are to perform a further heating process of multi-mode waveguide. In both cases the same initial diffusion processes were conducted: duration $t=1 \mathrm{~h}$, diffusion temperature $T=360^{\circ} \mathrm{C}$, admixture source $\mathrm{AgNO}_{3}$ in $\mathrm{NaNO}_{3}(z=0.0025 \mathrm{mf})$. In the first case the heating process was calculated for the parameters: $T=360^{\circ} \mathrm{C}, t=1 \mathrm{~h}$. In the second case the same temperature was preserved while increasing the heating time twice $t=2 \mathrm{~h}$. Both heating processes will decrease the refractive profile after the initial diffusion. At the same time the profile is expanded into the glass. This effect is obviously greater for a longer heating. The final step for both LPWG structures was secondary diffusion process: $t=1 \mathrm{~min}, T=330^{\circ} \mathrm{C}$, admixture source $\mathrm{AgNO}_{3}$ in $\mathrm{NaNO}_{3}$ solution $(z=0.0025 \mathrm{mf})$. This process produced a single-mode area of the LPWG structure.

Figure 7 presents the results of calculations for the simulations stated above. The final refractive index profiles of multi-mode areas are shown in Fig. 7a and c. These 

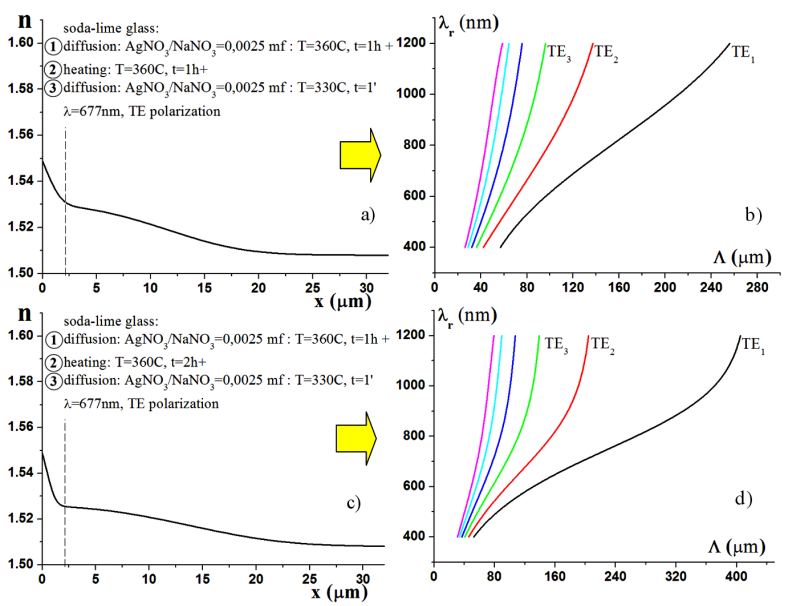

Fig. 7. Refractive index profiles $(\lambda=677 \mathrm{~nm}, \mathrm{TE})$ of LPWG structures produced in three technological stages: (a) and (c) and the resulting characteristics of resonant coupling of $\mathrm{TE}_{0}-\mathrm{TE}_{m}$ modes (b) and (d).

profiles are calculated for the wavelength $\lambda=677 \mathrm{~nm}$. The influence of doubled time of multi-mode waveguide's heating can be seen. Furthermore there is a greater difference between the increments in the refractive indices of the glass substrate. Next to Fig. $7 \mathrm{~b}$ and $\mathrm{d}$ the resonant characteristics $\lambda_{\mathrm{r}}(\Lambda)$ resulting from the refractive index profiles are shown. Attention is drawn here to the influence of heating on shifts in the characteristics in the corrugation period domain. The change is also to the shape characteristics, especially for the couplings of the low-order modes.

\subsection{Transmission spectra of $L P W G$ gradient-gradient structures}

The phenomenon of coupling modes in planar optical waveguides with the LPWG structures have been shown as the power coupling carried out in a basic $\mathrm{TE}_{0}$ mode to higher-order modes. This coupling has a resonant character. For a given LPWG structure with a $\Lambda$ period of corrugation there are strict wavelengths (2), for which there is conversion of power from the fundamental mode to higher order modes. In this case, there is a decrease of optical power conducted in the fundamental mode. By analyzing this phenomenon in the wavelengths domain the transmission characteristics are obtained for the fundamental mode (6). The coefficients of coupling modes $\kappa_{-1, \mathrm{TE}_{0} \rightarrow \mathrm{TE}_{m}}$ appearing in this Eq. (6) depend on the overlap integrals of the modal fields in the corrugation area (5). So a decisive influence on the magnitude of these coefficients has a depth $h$ of this corrugation. The changes in these coefficients depend on the wavelength of chromatic dispersion of mode fields.

Figure 8 shows a comparison of resonance characteristics for waveguide structures described previously. Next, the transmission characteristics are shown in a basic $\mathrm{TE}_{0}$ mode. In all cases, the same parameters of corrugated

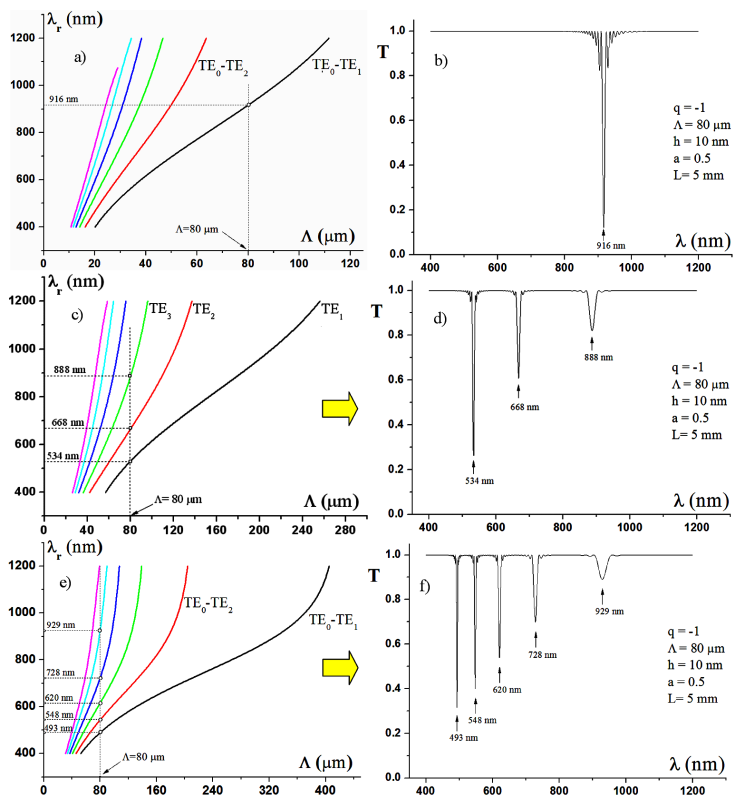

Fig. 8. The calculated resonance and transmission characteristics of LPWG structures for waveguides produced in the processes: (a) and (b) diffusion from solution $\mathrm{AgNO}_{3}$ in $\mathrm{NaNO}_{3}(z=0.0025 \mathrm{mf}): t=1 \mathrm{~h}$, $T=360{ }^{\circ} \mathrm{C}+$ diffusion $\mathrm{AgNO}_{3}: t=2 \min , T=260^{\circ} \mathrm{C}$, (c) and (d) diffusion from solution $\mathrm{AgNO}_{3}$ in $\mathrm{NaNO}_{3}$ $(z=0.0025 \mathrm{mf}): t=1 \mathrm{~h}, T=360^{\circ} \mathrm{C}+$ heating: $t=1 \mathrm{~h}, T=360^{\circ} \mathrm{C}+$ diffusion from solution $\mathrm{AgNO}_{3}$ in $\mathrm{NaNO}_{3}(z=0.0025 \mathrm{mf}): t=1 \mathrm{~min}, T=330^{\circ} \mathrm{C}$, (e) and (f) diffusion from solution $\mathrm{AgNO}_{3}$ in $\mathrm{NaNO}_{3}$ $(z=0.0025 \mathrm{mf}): t=1 \mathrm{~h}, T=360^{\circ} \mathrm{C}+$ heating: $t=2 \mathrm{~h}, T=360^{\circ} \mathrm{C}+$ diffusion from solution $\mathrm{AgNO}_{3}$ in $\mathrm{NaNO}_{3}(z=0.0025 \mathrm{mf}): t=1 \mathrm{~min}, T=330^{\circ} \mathrm{C}$.

area were assumed: $\Lambda=80 \mu \mathrm{m}, h=10 \mathrm{~nm}, a=0.5$ and $L=5 \mathrm{~mm}$. These characteristics show from one to five resonant couplings. The figure of merit of these resonances varies with the row of modes. The choice of parameters of corrugated area adopted here is not optimal. For each waveguide structure it should be decided individually. However, the purpose of this paper was to show the theoretical possibilities of producing LPWG structures on the basis of planar gradient waveguides.

\section{Conclusions}

This paper introduced the application possibilities of LPWG in planar structures of gradient waveguides. The element of novelty here is a proposal of gradient multi-mode decoupling area of modes in the corrugated area. The simulations of diffusion and heating processes for $\mathrm{Ag}^{+} \leftrightarrow \mathrm{Na}^{+}$ion exchange in soda-lime glass were carried out. The calculations included both: $\mathrm{AgNO}_{3}$ as well as $\mathrm{AgNO}_{3}$ solution in $\mathrm{NaNO}_{3}(z=0.0025 \mathrm{mf})$ as the admixture source. The actual dispersion of soda-lime glass refraction was also taken into account. For the waveguide area the assumption of constant refractive index increment (in relation to the glass substrate) was made 
in the whole analyzed spectral range. For the calculations, the exchange of binary diffusion model was used. This model assumes the dependence of diffusion coefficients of exchanged ions from their normalized concentration. The calculations used a set of experimental dependences of diffusion coefficients on temperature. This allowed the modeling of diffusion and heating processes at different temperatures. The simulations of producing waveguide LPWG structures in the processes of preliminary diffusion (multi-mode area) + secondary diffusion (single-mode waveguide) have been made. The calculations of simulating the process of heating the multi-mode area has been also done. The use of additional heating processes of the multi-mode area allows the usage of large corrugation periods $\Lambda$, for which the resonant coupling of $\mathrm{TE}_{0} \rightarrow \mathrm{TE}_{m}$ modes occur in the visible spectral range. For the chosen parameters of corrugation area the comparison was performed to compare the transmission characteristics in the basic $\mathrm{TE}_{0}$ mode for the three LPWG structures. These characteristics demonstrate the resonances consistent with calculations based on the properties of dispersion guided modes. The calculations show the possibilities of producing LPWG structures in planar waveguides systems with a gradient area of coupling modes.

\section{Acknowledgments}

This paper was financed from the funds of the $\mathrm{Na}$ tional Science Centre, awarded on the basis of the decision DEC-2011/01/B/ST7/06525.

\section{References}

[1] A.M. Vengsarkar, P.J. Lemaire, J.B. Judkins, V. Bhatia, T. Erdogan, J.E. Sipe, J. Lightwave Technol. 14, 58 (1996).

[2] P.J. Lemaire, R.M. Atkins, V. Mizrahi, W.A. Reed, Electron. Lett. 29, 1191 (1993)

[3] O. Duhem, A.DaCosta, J.F. Henninot, M. Douay, Electron. Lett. 35, 1014 (1999).
[4] C.Y. Lin, L.A. Wang, Photon. Technol. Lett. 13, 332 (2001).

[5] A.A. Abramov, A. Hale, R.S. Windeler, T.A. Strasser, Electron. Lett. 35, 81 (1999).

[6] H.J. Patrick, A.D. Kersey, F. Bucholtz, J. Lightwave Technol. 16, 1606 (1998).

[7] C.Y. Lin, G.W. Chern, L.A. Wang, J. Lightwave Technol. 19, 1212 (2001).

[8] Y. Liu, L. Zhang, I. Bennion, Electron. Lett. 35, 661 (1999).

[9] R. Rogoziński, P. Karasiński, Opto-Electron. Rev. 13, 229 (2005).

[10] H.C. Tsoi, W.H. Wong, E.Y.B. Pun, Photon. Technol. Lett. 15, 721 (2003).

[11] K.S. Chiang, K.P. Lor, C.K. Chow, H.P. Chan, V. Rastogi, Y.M. Chu, Photon. Technol. Lett. 15, 1094 (2003)

[12] V. Rastogi, K.S. Chiang, Appl. Opt. 41, 6351 (2002).

[13] S. Pal, B.R. Singh, J. Lightwave Technol. 25, 2260 (2007).

[14] Q. Liu, K.S. Chiang, K.P. Lor, Opt. Expr. 13, 1151 (2005).

[15] K.P. Lor, Q. Liu, K.S. Chiang, Photon. Technol. Lett. 17, 594 (2005).

[16] R. Rogoziński, Opt. Appl. 28, 331 (1998).

[17] A. Opilski, R. Rogoziński, K. Gut, M. Błahut, Z. Opilski, Opto-Electron. Rev. 8, 117 (2000).

[18] R. Rogoziński, Planar waveguide structures produced by ion exchange in glasses - Selected aspects of production technology, measurement of optical properties and numerical modelling of structures, Monograph 135, Wydawnictwo Politechniki Ślšskiej, Gliwice 2007 (in Polish)

[19] C. Chen, P. Berini, D. Feng, S. Tanev, V.P. Tzolov, Opt. Expr. 7, 260 (2000).

[20] D.-L. Zhang, Y. Zhang, Y-M.Cui, C-H.Chen, E.Y.B. Pun, Opt. Laser Technol. 39, 1204 (2007). 